\title{
A NOVEL ENF EXTRACTION APPROACH FOR REGION-OF-RECORDING IDENTIFICATION OF MEDIA RECORDINGS
}

\author{
Majed El Helou, Abdel Wahab Turkmani, Rawan Chanouha and \\ Samer Charbaji \\ Department of Electrical and Computer Engineering, \\ American University of Beirut, Beirut, Lebanon \\ $\{$ mhe33, ast11, rsc08, sac13\} @mail.aub.edu
}

\begin{abstract}
The electric network frequency (ENF) of power lines leaves its trace in nearby media recordings. The ENF signals vary in a consistent way in a given power grid. Therefore, it is possible to develop signal processing and machine learning techniques to identify the grid of origin by extracting attributes of the embedded ENF signal in recorded audio. This paper presents a model based on a novel ENF extraction technique with training on audio and power recordings from different grids. The proposed approach is based on correcting erroneously selected peaks from the Short Time Fourier Transform (STFT) by leveraging time correlations. These peaks are mistakenly taken for the frequency component belonging to the embedded ENF signal of the power grid and are corrected by the algorithm. Results on a test set of 50 recordings from nine different locations demonstrate the effectiveness of the proposed approach with an overall accuracy of $88 \%$.
\end{abstract}

\section{KEYWORDS}

ENF, Region of Recording, Media Recordings, Power Grids, Noise in Audio Recordings.

\section{INTRODUCTION}

Electric network frequency (ENF) signals, which are embedded in recordings made close to power lines, can be used to identify the origin of these media recordings. This serves the function of allowing audio recordings to be validated as unaltered in legal applications. Moreover, the uniqueness [1] of the embedded ENF components to it's grid of origin allow for the localisation of media recordings, allowing for applications in domains of authentication, security and crime fighting $[2,3]$.

State of the art methods involve examining the Fourier Transform over overlapping time frames of the data and combining identified frequency components, or their harmonics, to construct an estimate of the ENF signal variation, which is captured in the recording [4].

In this paper, a model for ENF extraction and grid of origin prediction is proposed. Particularly, the paper suggests a novel algorithm for improving the accuracy of the estimation of ENF values extracted from audio recordings. This algorithm comes as a solution to the presence of noise, which cannot be easily removed by using conventional noise cancellation algorithms since the David C. Wyld et al. (Eds) : DBDM, CICS, CSIP, AI\&FL, SCOM, CSE, CCNET-2016

DOI : $10.5121 /$ csit. 2016.60510 
ENF itself is considered as noise inside an audio recording. Particularly, the paper proposes a solution whereby correction is done by studying the strongest frequency component, checking if it satisfies certain noise thresholds, and then replacing it by the next strongest frequency component should it fail to do so.

In addition, the paper presents the design and implementation of a hardware circuit to capture power mains reference recordings. A simple circuit is used in tandem with an NI MyDAQ in order to record the reference signal. Finally, the data is analysed using our proposed algorithm and we show that our reference recordings belong to one of the recordings in the training set.

The rest of this paper is organised as follows. Section II covers some work done related to our topic. In Section III, details of the extraction of the electric network frequency signals from audio recordings and power reference recordings are discussed. Section IV tackles the details of the extraction of features of the ENF signal and discusses the machine-learning model generated. Section $\mathrm{V}$ covers the recording of local power reference signals and presents an analysis of the recordings. Section V concludes the paper.

\section{RELATED WORK}

The ENF signal constitutes a pattern observed in audio recordings. It represents the most significant frequency component of the voltage on a specific power grid at each second [18]. The analysis of ENF signals was motivated by many forensic applications. Multiple studies were conducted to explore the possibility of determining either the time of recording of the audio [19] or the location where it was recorded through the analysis of the ENF signal embedded in it [17]. Many means of extracting this signal were proposed in the literature. For example in [20], the authors divide the audio into overlapping frames and estimate the prevailing instantaneous frequency in each frame. However, in this paper, the authors focused on recaptured audio recordings which may contain multiple ENF signals. In [21], two main challenges faced in ENF estimation were tackled. The authors propose a special short time Fourier transform (STFT) approach to solve the issues of noise and frequency resolution. The latter is chosen to be equal to the threshold within which the true peak value can be recovered from the noise interference. Another algorithm for ENF extraction was discussed in [22]. The approach relies on binary search to narrow the search band until an accurate estimate of the ENF information is obtained. Another method proposed in [23] estimates the ENF signal by tracking it across different frames. The number of peaks in each frame is selected to minimize the Bayesian Information Criterion (BIC) model. Once the ENF signal is obtained, one needs to match it to other present signals in the database as presented in [23, 22, and 17]. In [17], it is proven that there exists an approximate linear relationship between the correlation coefficient between the observed ENF and a signal in the database and the distances between the two recording locations. In [23], this is done through minimizing a squared error and equivalently maximizing the correlation coefficient between the estimated ENF signal and the corresponding signal from the database. In [22], a penalty coefficient is introduced which will use conventional methods under good conditions (weak noise and long recording time) but adopt a different approach in case a non-trivial noise component is present by relying on the fact that the noise is not dominant during all time instances. In this paper, we present a novel method which relies on extracting features from the ENF signal and applying classical machine learning techniques in order to make an accurate guess on each of the extracted ENF signals.

\section{ENF EXTRACTION}

\subsection{Power grid operating frequency detection}

The ENF signal is defined as the sequence of the changing values over time of the instantaneous ENF, which is in itself the supply frequency of power distribution grids. The extraction of ENF 
begins with a close study of the Fast Fourier Transform (FFT) of the signals. Looking at the signal in the frequency domain, the aim was to find the component corresponding to the trace of the power grid, which is expected to be around $50 \mathrm{~Hz}$ or $60 \mathrm{~Hz}$, and then to estimate the exact instantaneous ENF of that grid.

The algorithm to extract the ENF signal across time starts by identifying whether the grid ENF is around $50 \mathrm{~Hz}$ or $60 \mathrm{~Hz}$. To achieve this, the proposed algorithm starts by taking the magnitude of the FFT of the recording. Since the recording is a real signal its FFT is even, and it is enough to study one of the two symmetric halves of the magnitude of the FFT. When studying the frequency components, it is noticeable that in some signals some harmonics of the $50 \mathrm{~Hz}$ or $60 \mathrm{~Hz}$ component are relatively stronger than others, whereas some are relatively very weak. Because most harmonics were negligible compared to the strongest one in the majority of cases, and mostly to keep the complexity minimal, the algorithm considers the ENF at the strongest harmonic

The algorithm computes the distance between the highest frequency component $\mathrm{F}$ in the FFT and each of the first 20 harmonics of 50/60Hz (For ex: 50,100, 150,200Hz etc. and 60, 120, 180, $240 \mathrm{~Hz}$ etc.). The closest harmonic to the strongest frequency value is thus determined. Let $\mathrm{d}$ be the distance between that harmonic and $\mathrm{F}$. This closest harmonic, $\mathrm{H}_{\text {number }}$, is taken as the correct one corresponding to the power grid if $d$ is less than a chosen frequency filter half width $F_{w / 2}$ (half the width of a rectangular window filter chosen to be applied in the frequency domain, which will be discussed shortly). The width of the window is always scaled to the corresponding harmonic. This procedure can be expressed as follows:

$$
\mathrm{d}<\mathrm{F}_{\mathrm{w} / 2} * \mathrm{H}_{\text {number }}
$$

\subsection{ENF Estimation}

After determining whether the grid ENF is around $50 \mathrm{~Hz}$ or $60 \mathrm{~Hz}$, the algorithm creates the aforementioned bandpass filter centred at F:

$$
\left(F-F_{w / 2}, F+F_{w / 2}\right) H z
$$

Once the filter is created, the algorithm computes a short-time Fourier Transform (STFT). This is done by first choosing a padding factor $(\mathrm{P})$, time window $(\mathrm{Tw}(\mathrm{sec})$ and a per cent overlap $(\mathrm{O})$ then moving in time along the recording. The FFT is taken across consecutive sections of the recording, after padding the section with zeros to increase the length in time of each section by the padding factor and thus have a more precise frequency domain signal after taking the FFT [5, 6]. The samplinglfrequency of all the down-sampled signals used being $1 \mathrm{kHz}$, the FFT bin size is equal to:

$$
0.5 \log 2(T w * P * 1 k H z)
$$

Each section will have a width equal to Tw seconds, and will be overlapping with the previous section over a duration of time equal to $\mathrm{Tw} * \mathrm{O}$ seconds.

The bandpass filter is applied in the frequency domain to the consecutive FFT signals computed on these time windows to get rid of as much noise as possible [7].

From the output of the filter, the strongest frequency component is determined. Using this frequency value as well as the one right before it and the one right after it (the two adjacent FFT frequency bins) the algorithm estimates the value of the strongest frequency component by quadratic interpolation $[5,8,9]$ which makes up for the precision loss of the discrete Fourier transform. 
Finally, the ENF signal is scaled back to the base harmonic by regular division in case it was extracted from a higher order harmonic, simply for the conservation of consistency in all readings.

\subsection{Peak Correction in noisy signals}

At this stage, the ENF signals extracted from power recordings are in conformity with ENF signals seen in the literature [10]. However, those extracted from audio signals remain highly noisy i.e. having many fast variations above and below the moving mean of the ENF, in comparison with the ENF signals extracted from power recordings taken from the same power grid. Simple smoothing algorithms, including median filtering [8] and outlier rejection algorithms do not fix the incorrect readings in addition to making the initially accurate readings deviate from the correct frequency values.

Looking carefully at the frequency domain equivalent of time windows where readings that are deemed incorrect and that cause a noisy ENF were taking place, the following was observed: in the frequency domain, the strongest frequency component around the chosen harmonic is correctly identified, however, the problem is this peak is not in fact the ENF component, but simply a noise frequency [4]. As can be seen in the Figure 1, there is another strong local peak inside the bandpass filter region, which is slightly weaker than the highest peak first found.

The two border labels in the figure delimit the bandpass filter frequency limits, while the two middle labelled points correspond to the local peaks mentioned above.

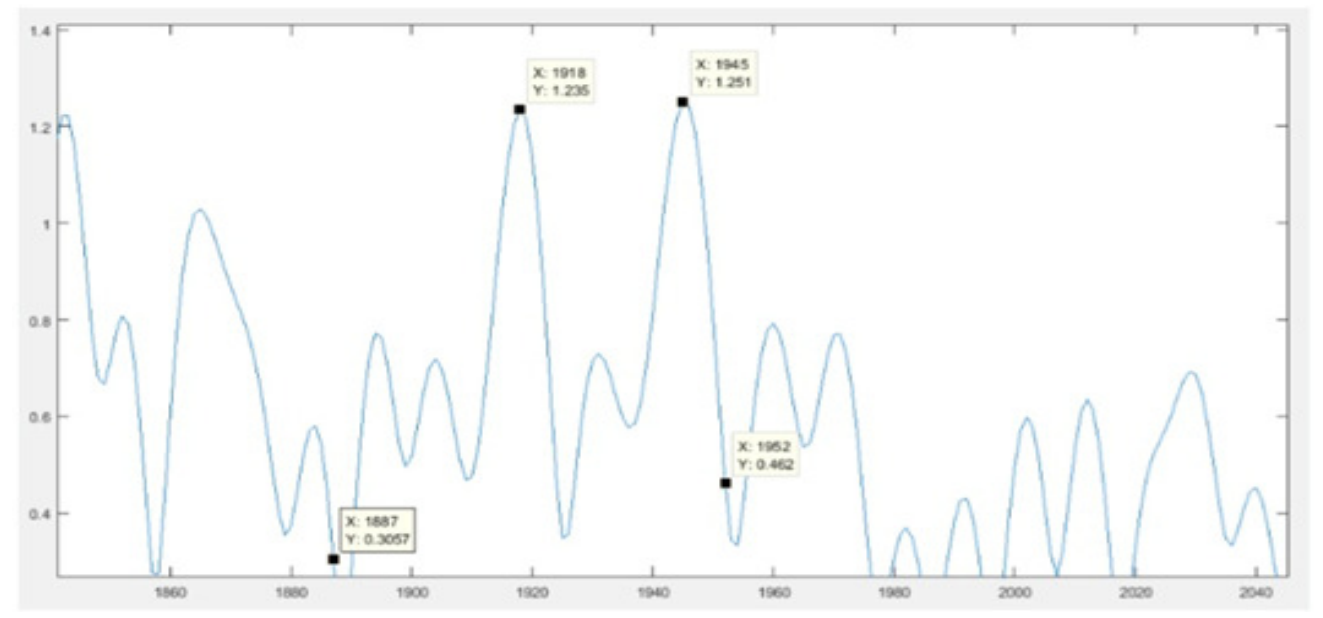

Figure 1. Magnitude vs. Frequency in the FFT of a Time Window

The global peak $\left(\mathrm{P}_{\mathrm{G}}\right)$ at frequency $\mathrm{F}_{\mathrm{G}}$, which causes the extracted ENF to be noisy, is very close to the filter's upper limit, while the next local peak $\left(\mathrm{P}_{2}\right)$ located at frequency $\mathrm{F}_{2}$ is centred in the middle of the bandpass filter. Through the inspection of many similar cases of noisy points in the final ENF, the conclusion that this second largest local peak inside the bandpass filter is actually the ENF component was drawn. This conclusion holds in a wide majority of the noise cases. An algorithm was developed to identify these second strongest local peaks while extracting the ENF signal. Imposing a noise threshold $\left(\mathrm{N}_{\mathrm{o}}\right)$ and considering the previously computed instantaneous ENF value $\mathrm{F}_{\text {old }}$, if the conditions (4) and (5) hold, then the second local peak is taken to be the ENF component and not the global peak.

$$
\mathrm{P} 2>\mathrm{No} * \mathrm{PG}
$$



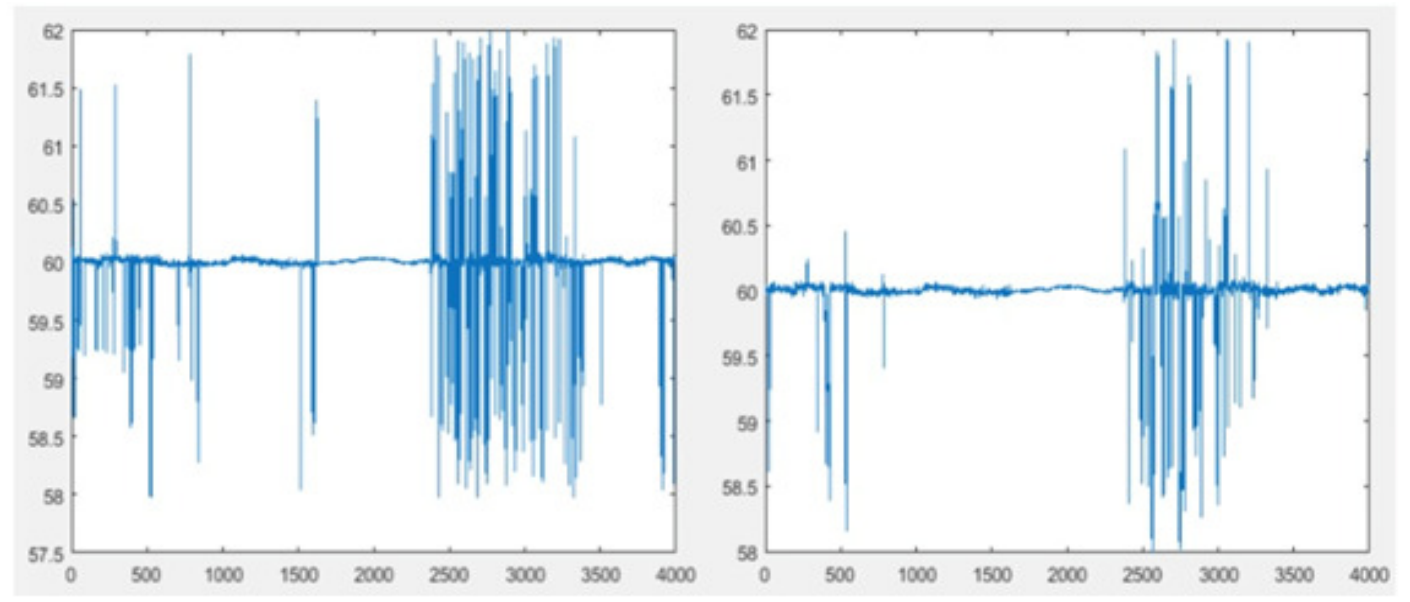

Figure 2. Audio ENF Post-Processing

Figure 2 shows the extracted ENF without applying the novel post-processing detection algorithm (left figure) and the ENF extracted with the post-processing algorithm applied (right figure). As can be noted, the right figure ENF has significantly less noisy points than the ENF extracted without the novel algorithm, and, even more importantly, points that are not originally noisy are not affected. There remains a fraction of the noisy point readings in the right figure for the following reasons:

1) The algorithm is not applied very aggressively by setting the noise threshold to relatively high percentage values. This ensures that the algorithm does not affect previously correctly predicted ENF values. Thus correct estimates remain unaffected with high probability.

2) Additionally, the algorithm only accounted here for the second strongest peak and corrected between that peak and the global maximum peak. However, it is possible to concurrently consider the third, fourth, etc. peaks in correcting the original one. Nevertheless, this comes at a higher computational cost.

The algorithm leverages the fact that there is an inherent time correlation between consecutive estimated instantaneous ENF values, and thus the variability in ENF values cannot be, on average, too large. This is because the ENF of the grid at time $t_{n}$ is not independent from that at time $t_{n-1}$, the distribution could actually be approximated by a random walk if the variations on the grid were assumed to be Gaussian noise. The algorithm thus takes advantage of that information to single out estimations that are altered by noise and estimates again a more probabilistically plausible value of the ENF at that time instant.

Since the problem at hand does not occur in the case of power recordings, this extra processing is only done for audio recordings to reduce the running time. To differentiate whether a given recorded signal is a power recording or an audio recording, the power spectral density (PSD) of the signal is compared to a chosen threshold: PSD threshold. The power spectral density of a power recording is far larger than that of an audio recording because the power is highly focused at the ENF component only, unlike with audio recordings. Audio recordings contain many more frequency components aside from the ENF, the latter being simply a trace. This makes this decision accurate and highly confident. 
Finally, all of the aforementioned parameters were chosen after thorough testing and studying of the processing steps as well as the literature (for first four parameters) and are grouped in Table 1.

The frequency filter half width $\left(F_{w / 2}\right)$ was set to $2 \mathrm{~Hz}$ at the base harmonic to filter out as much noise as possible (the ENF signals not having a range going beyond $4 \mathrm{~Hz}$ ), while not affecting the frequency components inside the bandpass filter by making it too narrow, since no rectangular filter in the frequency domain is ideal as its counterpart requires infinite length in the time domain (infinite sinc function) [11].

The time window $\left(T_{w}\right)$ was initially chosen to be 5 seconds [10] but was then reduced to only 3 seconds through testing to achieve higher accuracies. Additionally shorter window times result in a better fit with the autoregressive model, which would fit a shorter signal in general more accurately than a lengthier one. The same was done with the per cent overlap $(O)$ which, initially set to be $50 \%$, ended up being an $85 \%$ overlap after tweaking. For the padding factor, the chosen value was based on the observations from the maximum-bias curves for "rect" windows noted in [5], in a way to reduce the final bias ratio. Lastly, the noise threshold $\left(\mathrm{N}_{\mathrm{O}}\right)$ of the newly proposed algorithm was first chosen by inspection of cases similar to Figure 1, and then tweaked for best model accuracy.

Table 1. Parameter Values of the Proposed Model

\begin{tabular}{|l|l|}
\hline Parameter & Chosen Value \\
\hline frequency filter half width & $2 \mathrm{~Hz}$ \\
\hline time window & 3 seconds \\
\hline padding factor & 16 \\
\hline percent overlap & $85 \%$ \\
\hline noise threshold & 0.7 \\
\hline
\end{tabular}

\section{ORIGIN CLASSIFICATION}

\subsection{Selected Features}

After careful examination of the extracted ENF signals, a list of features were identified in order to differentiate between the signals coming from different grids by training a machine learning model on those features extracted from training data. The first feature chosen is the mean of the ENF signal. As explained in [10], the mean not only allows us to identify whether the signal belongs to a $50 \mathrm{~Hz}$ grid or a $60 \mathrm{~Hz}$ grid but also within the same type of grids the mean is never exactly equal to its ideal value and this shift from the ideal frequency is reasonably consistent in the recordings within the same grid.

Other important statistical measures chosen are the variance, the median and the mode, which are additional descriptors of the ENF signal, noted to be rather consistent for a given grid. The range as well as the minimum and the maximum values of the ENF have also proven to provide a useful insight for the classification process by helping with the distinction between some grids. A log base 10 operator is applied to the range and the variance instead of directly using their initial values to emphasize the fact that only a significant change in these parameters is wanted to affect the classification model [10]. The skewness, which is defined as a measure of the degree of symmetry in the data, and the kurtosis, describing the sharpness of the peaks near the mean, both were used as features in classifying the recordings, with the kurtosis being a notably relevant feature characterizing ENF signals coming from different grids. 
In addition, a wavelet transform is applied to the original ENF signal in order to extract statistical characteristics of the data at different resolution levels [10]. Specifically, a 9-Level wavelet decomposition was chosen and the log base 10 of the variance of each of the 9 resulting detailed levels was used as a statistical feature from every resolution level. Also the variance of the approximated signal is used to reflect the general variation pattern of the signal ignoring relatively high- frequency variations in the ENF signals. An autoregressive (AR) model, of order 2 , was then developed.

$$
y[n]=x 1 y[n-1]+x 2 y[n-2]+v[n]
$$

Three features were obtained from this new model, notably the two AR parameters as well as the $\log$ base 10 of the variance of the model. These features give insight on how the signal can be fitted into an AR model [10].

Additionally, spectrum features are also famous parameters used in audio analysis. Among these features, the spectral centroid, a measure of the centre of gravity of the STFT' s magnitude [12], and the spectral roll-off, which represents the frequency below which a fraction (commonly $85 \%$ ) of the total energy is present, proved to give better results in terms of the accuracy of the classification when integrated into the model. The mean crossing was also computed to capture how many times the ENF signal crosses its mean value, going over and under it, and also, the zero-crossings of the derivative of the ENF signal as well the maximum value of the derivative were added as classification features to portray how fast the ENF signal changes its direction of variation (increasing, decreasing) and how abrupt is its fastest change in frequency value.

Finally, the outlier ratio of the ENF signal, which is a measure of instances that do not fall within two standard deviations of the mean value, also turned out to be a suitable criterion for the classification. These 28 features, mentioned earlier, constitute the training set for the classifier and are summarized in the Table 2 .

Additionally all the features which are affected by ENF signal length were normalized by dividing their value by the signal's length, although all training data was finally chosen to have equal length of 20 minutes per sample as explained shortly.

Table 2. Summary of Selected Features

\begin{tabular}{|c|c|}
\hline Index & Feature \\
\hline 1 & Mean of ENF signal. \\
\hline 2 & Log10 (var) of ENF signal. \\
\hline 3 & Log10 (range) of ENF signal. \\
\hline $4-12$ & $\begin{array}{l}\text { Log } 10 \text { (var) of the } 9 \text { detailed levels of the ENF } \\
\text { signal obtained by applying a wavelet transform } \\
\text { on the original signal. }\end{array}$ \\
\hline 13 & $\begin{array}{l}\text { Log } 10 \text { (var) of the approximated signal obtained } \\
\text { after applying the wavelet transform (with } 9 \\
\text { levels) on the original ENF. }\end{array}$ \\
\hline $14-15$ & Auto-regression parameters. \\
\hline 16 & $\begin{array}{l}\text { Log10 (var) of the autoregressive model } \\
\text { innovation signal. }\end{array}$ \\
\hline
\end{tabular}




\begin{tabular}{|c|l|}
\hline 17 & Median of the ENF signal. \\
\hline 18 & Mode of the ENF signal. \\
\hline 19 & Skewness of the ENF signal. \\
\hline 20 & Kurtosis of the ENF signal. \\
\hline $21-22$ & $\begin{array}{l}\text { The minimum and maximum value of the ENF } \\
\text { signal respectively. }\end{array}$ \\
\hline 23 & Mean crossing of the ENF signal. \\
\hline $24-25$ & $\begin{array}{l}\text { Spectral centroid and spectral roll-off } \\
\text { respectively. }\end{array}$ \\
\hline 26 & Maximum derivative. \\
\hline 27 & Outlier ratio. \\
\hline 28 & Derivative zero-crossing. \\
\hline
\end{tabular}

\subsection{Classification Model}

Prior to training the classifier, the training data was split into 20-minute frames, with a $50 \%$ overlap across frames. This increased the total training set from 111 to 790 entries and contributed substantially to increasing the accuracy of the classifier. Among all the classifiers tested, Random Forests have proven to be the most successful. They outperformed SVM, AdaBoost, and many other widely used algorithms. This is due to the inherent randomness in selecting the data to train its weak learners on, and in selecting the features to provide its weak learners (decision trees) to split on [13].

These characteristics, combined with splitting the training data into overlapping smaller components, which resulted in large quantities of data samples to train on, ensued in remarkable gains in accuracy. The chosen bagging tree classifier is trained over 30 cycles, and actually 255 such classifiers are generated. Each of the 255 classifiers is trained to predict whether or not a sample belongs to a chosen subset of the 9 grid classes, and for prediction, all of the 255 classification results are combined into a single one. There are 255 possible subsets from the 9 grids, 255 being $2^{\# \text { of grids - } 1}-1(-1$ because the subset containing all of the 9 grids is meaningless).

It is noteworthy to mention that the trained classifier does not account for a none-of-theabove option to keep the focus exclusively on classifying samples belonging to grids the classifier was trained on as previous works have shown this approach to actually improve overall accuracy [10]. Lastly, the classification model is used to generate posterior probabilities corresponding to each grid class, which are used as confidence measures in every prediction.

The proposed algorithm was tested on a test dataset with 50 recordings, comprising a combination of audio and power recordings coming from more than the 9 grids in the training set. As mentioned above, the constructed model does not account for out-oftraining- set $(\mathrm{N})$ classes and thus will classify them erroneously. Tables 3 and 4 contain the performance results of the full model on the test dataset. Table 3 portrays the accuracy of the model without using the novel ENF estimation algorithm in noisy audio recordings. 
Table 3 Result of Classification without Applying the Novel Peak-Correction Algorithm

\begin{tabular}{|c|c|c|c|}
\hline Accuracy (\%) & Power & Audio & Power and Audio \\
\hline Including N & 89.2857 & 54.5454 & 74 \\
\hline
\end{tabular}

This accuracy clearly improves with the application of the peak-correction algorithm, through significantly improving the system's performance on audio recordings which are naturally more noisy signals. Final accuracies are reported in Table 4.

With the first row indicating the accuracies when the signals belonging to class $\mathrm{N}$ (out of training set class) are included and the second row indicating the accuracy only on signals belonging to classes the model was trained on. As can be seen, the algorithm performs exceptionally well in predicting the origin of signals it was trained on, with the accuracy of audio signals displaying a tremendous improvement using the novel peak-correction technique.

Table 4. Results of Classification on Test Dataset with the Novel Algorithm

\begin{tabular}{|c|c|c|c|}
\hline Accuracy (\%) & Power & Audio & Power and Audio \\
\hline Excluding N & 96.4285 & 100 & 98 \\
\hline Including N & 89.2857 & 86.3636 & 88 \\
\hline
\end{tabular}

\section{DATA ACQUISITION AND ANALYSIS}

To obtain reference power recordings, a simple circuit, shown in the diagram below, was devised based on the one used in [14]. The focus in this circuit is on its simplicity and its portable design as well as on the accessibility of its components, which consist of a transformer, operational amplifier, capacitor, and resistors. The circuit is easily portable and can be directly plugged into different wall outlets in any location, which makes collecting recordings at different locations within the grid a quite simple task.

The step down transformer is used to bring down the 220VAC signal from the power outlet to a $25 \mathrm{~V}$ peak-to- peak signal. The signal is then passed through a low pass filter, which consists of a simple RC circuit with the output signal taken across the capacitor. The RC circuit serves as a low-pass filter and performs anti-aliasing to allow for proper acquisition. In order to remove the loading effect and further bring down the voltage to readable values, a unity gain amplifier with a voltage divider at the buffer output is utilised, resulting in a final $2 \mathrm{~V}$ peak-to-peak signal biased at $0 \mathrm{~V}$ [15]. The resulting output signal is read by a NI MyDAQ hardware and fed into LabVIEW where it is recorded at a sampling rate of 1000 samples/sec and number of samples equal to 1000 . The resulting signal is then exported as a .wav file to the machine running LabVIEW.

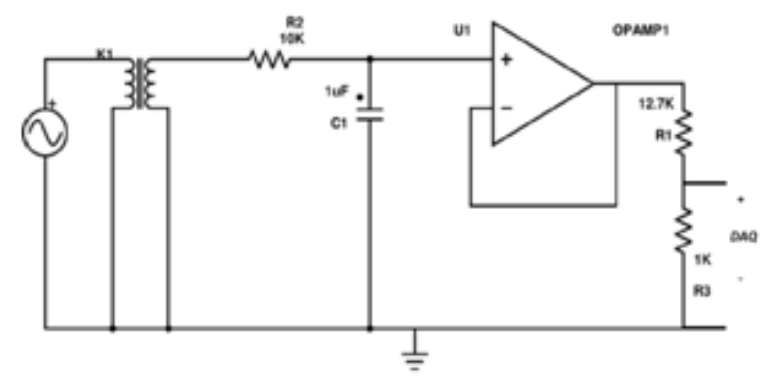

Figure 3 Schematic of the Chosen Circuit Design. 
Over the duration of our work, we collected nigh onto 14 hours, in one-hour samples, of clean power recordings from the power grid in Lebanon. Much care was taken to filter out recordings where mains power was cut due to country-wide regular power outages and instead back-up generators were used to supply electricity. Next, the ENF component of the signals is extracted, and a sample is shown in Figure 5.

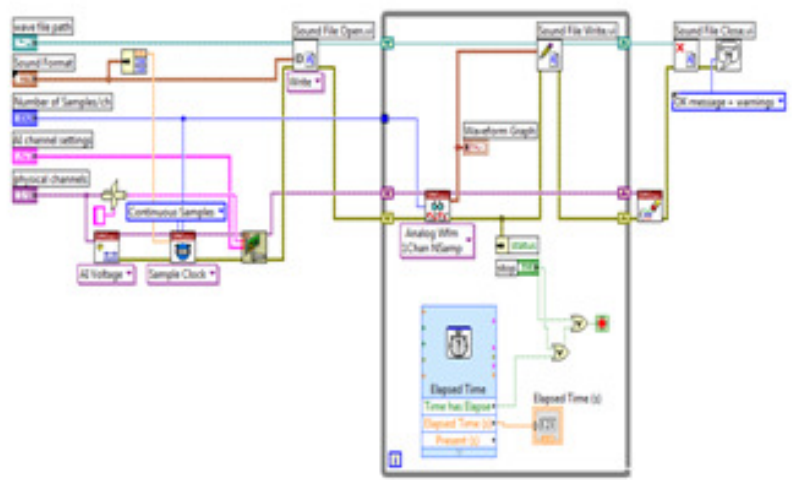

Figure 4 LabVIEW Data Acquisition VI.

Across all the acquisitions, it can be noted that on average the ENF was centred around $50.6 \mathrm{~Hz}$ to $50.8 \mathrm{~Hz}$. Additionally, a common trend observed across recordings are occasional plunges of magnitude around $1 \mathrm{~Hz}$ to $1.5 \mathrm{~Hz}$ in the ENF, with drops from $\sim 50.7 \mathrm{~Hz}$ down to $49.3 \mathrm{~Hz}$ to be the trend. A sample ENF is shown in Figure 5, highlighting one of the plunges.

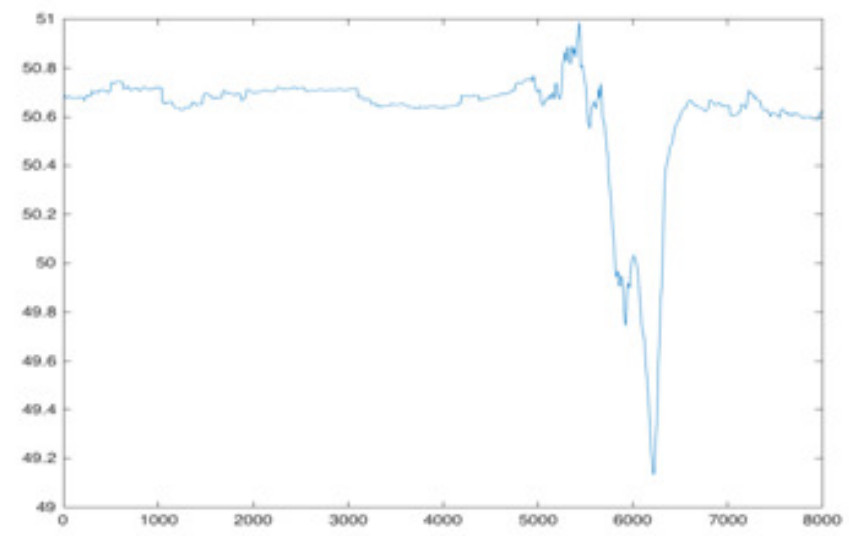

Figure 5 ENF of Signal Recorded using MyDAQ.

\section{CONClusions}

In this paper, we document our work for building a model for the extraction of ENF from media signals and for the classification of the origin of media recordings and power mains reference signal recording. Additionally, we propose a novel technique for ENF extraction that considerably improves the accuracy of the extraction in the presence of noise, as indicated by the difference in accuracies obtained with and without the ENF extraction method and as portrayed in the discussions. This method leverage the existence of correlation across time between ENF values, and thus estimates instantaneous ENF values with aim a reduction in overall variability frequency, thus having a similar yet much more accurate effect than smoothing. Moreover, we present the multi-class machine learning algorithm utilized to classify each grid as well as the features that were employed in the classification model. Lastly, for the purpose of ensuring reproducibility the hardware system developed is also portrayed in the paper. 


\section{REFERENCES}

[1] M. H. Bollen and I. Gu, "Signal processing of Power Quality Disturbances," Hoboken, NH, USA: Wiley, 2006.

[2] C. Grigoras, "Applications of ENF Analysis in Forensic Authentication of Digital Audio and Video Recordings,” J. Audio Engineering Society, vol. 57, no. 9,.pp. 643-66. Sept. 2009

[3] N. Fechner and M. Kirchner, "The Humming Hum: Background Noise as a Carrier of ENF Artifacts in Mobile Device Audio Recordings," in Proc. Of 2014 8th International Conference on IT Security Incident Management \& IT Forensics, Munster, Germany, pp. 3-13. May 2014,

[4] A. Hajj-Ahmad, R. Garg and M. Wu, "ENF-Based Region-of-Recording Identification for Media Signals", in Proc. Of IEEE Trans.Inform.Forensic Secur., vol. 10, no. 6, pp. 1125-1136, 2015.

[5] M. Abe, \& J.O Smith III, "Design criteria for simple sinusoidal parameter estimation based on quadratic interpolation of FFT magnitude peaks." In Audio Engineering Society Convention 117. Audio Engineering Society 2004.

[6] A. Cooper, "An automated approach to the Electric Network Frequency (ENF) criterion - Theory and practice", IJSLL, vol. 16, no. 2, 2010.

[7] J. O. Smith and X.Serra, "PARSHL: An analysis/synthesis program for nonharmonic sounds based on sinusoidal representation,” in Proc. Of Int. Computer Music Conf. pp. 290-297. 1987

[8] A. Hajj-Ahmad, R. Garg and M. Wu, "Spectrum Combining for ENF Signal Estimation", in Proc. Of IEEE Signal Processing Letters, vol. 20, no. 9, pp. 885-888, 2013.

[9] O. Ojowu, J. Karlsson, J. Li and Y. Liu, "ENF Extraction From Digital Recordings Using Adaptive Techniques and Frequency Tracking", in Proc. Of IEEE Trans.Inform.Forensic Secur., vol. 7, no. 4, pp. 1330-1338, 2012.

[10] Y. Yang and H. Chen, Music emotion recognition. Boca Raton, Fla.: CRC, pp.44-45, 2011.

[11] Y. Zhang and D. LV, "Selected Features for Classifying Environmental Audio Data with Random Forest", TOAUTOCJ, vol. 7, no. 1, pp. 135-142, 2015.

[12] P. Top, M.R. Bell, E. Coyle, O. Wasynczuk, "Observing the Power Grid: Working Toward a More Intelligent, Efficient, and Reliable Smart Grid with Increasing User Visibility," in Proc. Of Signal Processing Magazine, IEEE, vol.29, no.5, pp.24-32, Sept. 2012

[13] Y. Liu; C. Yao; C. Sun; Y. Liu, "The Authentication of Digital Audio Recordings Using Power System Frequency," in Proc. Of Potentials, IEEE , vol.33, no.2, pp.39-42, March-April 2014

[14] T. Yardibi, J. Li, P. Stoica, M. Xue, and A. B. Baggeroer, "Source localization and sensing: A nonparametric iterative adaptive approach based on weighted least squares," in Proc. Of IEEE Trans. Aerospace Electron. Syst., vol. 46, no. 1, pp. 425-443,Jan.2010.

[15] B. Kosko, Noise. New York: Viking, 2006

[16] M. Stamm, Min Wu and K. Liu, "Information Forensics: An Overview of the First Decade", IEEE Access, vol. 1, pp. 167-200,2013.

[17] R. Garg, A. Hajj-Ahmad and M. Wu , "Geo-location estimation from electrical network frequency signals" , in Proc. IEEE Int. Conf. Acoust., Speech, Signal Process. , pp.1 -3

[18] T. Baksteen, "The ENF criterion: A literature study", 2015. 
[19] M. Huijbregtse and Z. Geradts, "Using the ENF criterion for determining the time of recording of short digital audio recordings", Computational Forensics, pp. 116-124, 2009.

[20] H. Su, R. Garg, A. Hajj-Ahmad and M. Wu, "ENF analysis on recaptured audio recordings", Acoustics, Speech and Signal Processing (ICASSP), 2013 IEEE International Conference on,, pp. 3018-3022, 2013.

[21] G. Hua, J. Goh and V. Thing, "A Dynamic Matching Algorithm for Audio Timestamp Identification Using the ENF Criterion", IEEE Trans.Inform.Forensic Secur., vol. 9, no. 7, pp. 1045-1055, 2014.

[22] Ling Fu, P. Markham, R. Conners and Yilu Liu, "An Improved Discrete Fourier Transform-Based Algorithm for Electric Network Frequency Extraction", IEEE Trans.Inform.Forensic Secur., vol. 8, no. 7, pp. 1173-1181, 2013.

[23] O. Ojowu, J. Karlsson, J. Li and Y. Liu, "ENF Extraction From Digital Recordings Using Adaptive Techniques and Frequency Tracking", IEEE Trans.Inform.Forensic Secur., vol. 7, no. 4, pp. 13301338, 2012.

\section{AUTHORS}

Majed El Helou is currently pursuing a B.E. degree in computer and communication engineering with a math minor from the American University of Beirut, Beirut, Lebanon, expected to graduate in 2016. He was with the Computer Science and Artificial Intelligence Lab at the Massachusetts Institute of Technology as a Research Intern in the Wireless Net group, in summer 2015. His current research interests include signal and image processing, machine learning and statistical analysis, biomedical engineering and communication. He was listed on the Faculty of Engineering and Architecture Dean's Honor List for every semester of his undergraduate studies.

Abdel Wahab Turkmani is currently pursuing a B.E. degree in electrical and computer engineering from the American University of Beirut, Beirut, Lebanon, expected to graduate in 2016. He was with the Applications Engineering team at National Instruments as an Electrical Engineering Intern during the summer of 2015. His current research interests include machine learning, statistical analysis, computer vision and computational finance. He was listed on the Faculty of Engineering and Architecture Dean's Honor List for every semester of his undergraduate studies.

Rawan Chanouha is currently pursuing a B.E. degree in electrical and computer engineering with a math minor from the American University of Beirut, Beirut, Lebanon, expected to graduate in 2016. She was with the electrical engineering department at Veolia Water Technologies as an Electrical Engineering Intern, in summer 2015. Her current research interests include machine learning, big data, antenna design and biomedical engineering. She was listed on the Faculty of Engineering and Architecture Dean's Honor List for every semester of her undergraduate studies.

Samer Charbaji is currently pursuing a B.E. degree in computer and communications engineering from the American University of Beirut, Beirut, Lebanon, expected to graduate in 2016. He was with the Information Visualization and Management Lab in Purdue Polytechnic as a Research Intern, in summer 2015. His current research interests include machine learning, big data, supply chain management and strategic IT adoption. He was listed on the Faculty of Engineering and Architecture Dean's Honor List for every semester of his undergraduate studies. 